\title{
Prescribing Habits for Androgenic Alopecia among Dermatologists in Spain in 2019-2020: A Cross-Sectional Study
}

\author{
Cristina Pindado-Ortega ${ }^{a}$ b David Saceda-Corralo ${ }^{a, b}$ Diego Fernández-Nieto ${ }^{a}$ \\ Juan Jiménez-Cauhéa Daniel Ortega-Quijano ${ }^{a}$ Óscar M. Moreno-Arrones ${ }^{a}{ }^{\mathrm{b}}$ \\ Sergio Vañó-Galván a, b \\ aDermatology Department, University Hospital Ramón y Cajal, Madrid, Spain; ${ }^{b}$ Pedro Jaén Dermatology Group, \\ Madrid, Spain
}

\section{Keywords}

Hair · Hair loss · Minoxidil · Dutasteride; Pattern hair loss

\begin{abstract}
Background: In 2017, we carried out a cross-sectional study with the aim of describing androgenic alopecia (AGA) prescription habits among dermatologists who practice in Spain. Three years later, we repeated this study with the aim of describing the current situation and comparing it with previous data. Material and Methods: Descriptive cross-sectional study using online questionnaires autocompleted by dermatologists working in Spain. Results: The responses of 243 dermatologists were analyzed. The most common treatments prescribed for MAGA were topical minoxidil (100\%), oral finasteride (92.6\%), oral dutasteride (75.7\%), and oral minoxidil (50.6\%). For premenopausal female AGA (FAGA), the most common treatments were topical minoxidil (99\%), oral contraceptives (75\%), nutricosmetics (71\%), and oral minoxidil (67.9\%). Lastly, the most common treatments prescribed in patients with postmenopausal FAGA were topical minoxidil (99.2\%), oral finasteride (79\%), oral durasteride (71.6\%), and oral minoxidil (63\%). Conclusions: In conclusion, AGA is the most frequent alopecia in the trichology clinic. The most
\end{abstract}

common treatments prescribed in male AGA (MAGA) and postmenopausal FAGA were topical minoxidil and oral finasteride, while in premenopausal FAGA, topical minoxidil, oral contraceptives, and nutricosmetics were most commonly prescribed. In the past three years, oral dutasteride and oral minoxidil have increased drastically among the most prescribed therapies for MAGA, premenopausal FAGA, and postmenopausal FAGA.

(c) 2020 S. Karger AG, Basel

\section{Introduction}

Over the last few years, new therapies have been incorporated into the therapeutic arsenal against androgenic alopecia (AGA). In 2017, we carried out a cross-sectional study with the aim of describing AGA prescribing habits among dermatologists who practice in Spain [1]. Three years later, we repeated this study with the aim of describing the current situation and comparing it with previous data. For this purpose, a descriptive cross-sectional study was designed using a digital questionnaire exclusively addressed to dermatologists practicing in the Spanish territory, conducted between December 2019 and January 2020.

$\begin{aligned} & \text { karger@karger.com } \\ & \text { www.karger.com/sad }\end{aligned}$
Karger ${ }^{\prime /}$

Cristina Pindado-Ortega

Dermatology Department, University Hospital Ramón y Cajal Carretera Colmenar Viejo km 9.100

ES-28034 Madrid (Spain)

cpindadoortega@gmail.com 
Fig. 1. Frequency of each treatment in patients with male androgenic alopecia (blue bar: public activity; orange bar: private activity). PRP, platelet-rich plasma.
Fig. 2. Frequency of each treatment in patients with premenopausal female androgenic alopecia (blue bar: public activity; orange bar: private activity). PRP, plateletrich plasma.
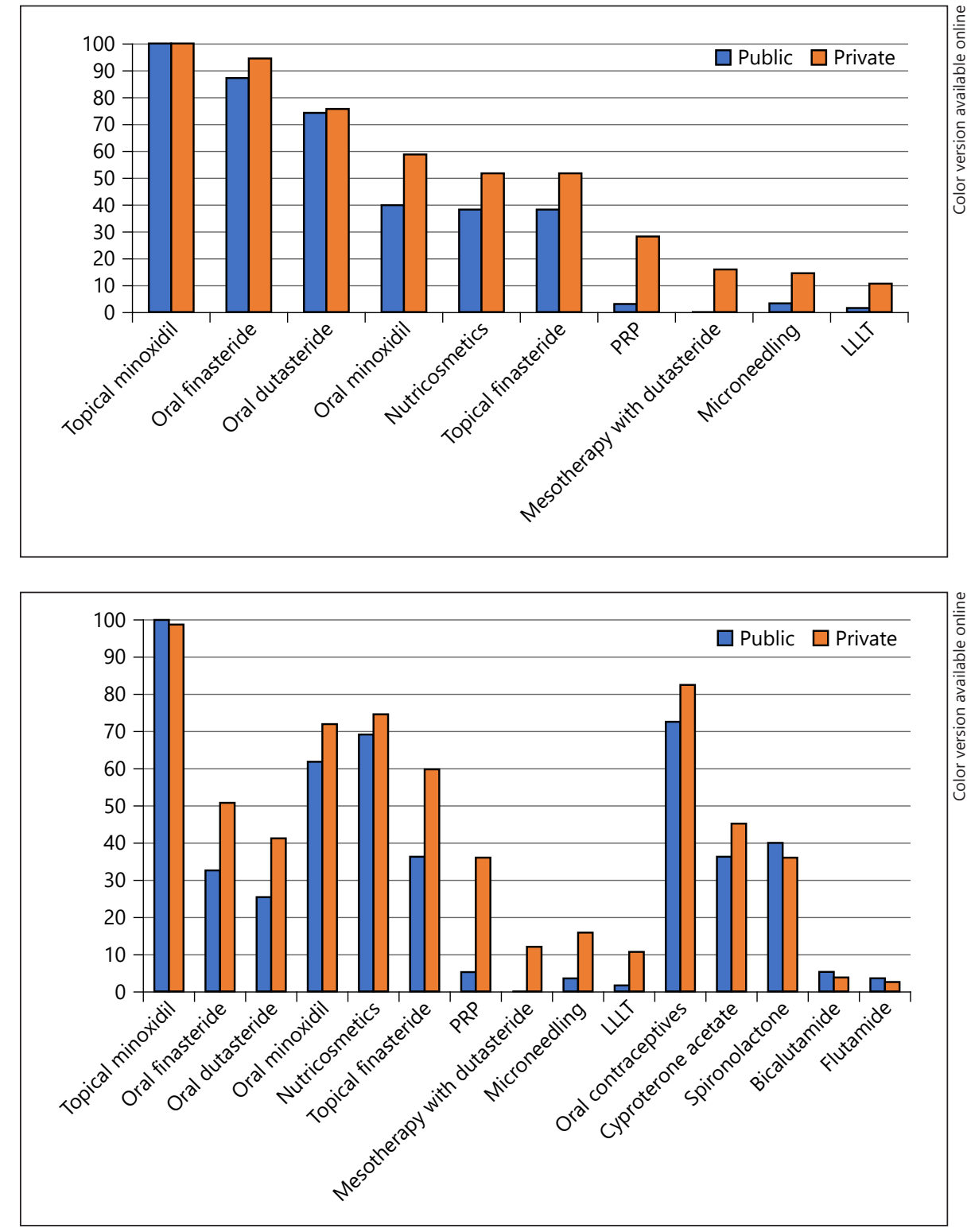

\section{Materials and Methods}

The responses of a total of 243 dermatologists were included; of them, $192(79.0 \%)$ were medical doctors with general consultation, 20 (8.2\%) medical doctors with a specialized trichology consultation, and 31 (12.8\%) dermatology residents.

Regarding the workplace, 75 dermatologists (30.9\%) carried out their work in a private consultation with a median of 12 trichology patients per week $\left(\mathrm{P}_{25}-\mathrm{P}_{75}=8.0-20.0\right), 55$ dermatologists $(22.6 \%)$ worked in the public system with a median of 10 trichology patients per week $\left(\mathrm{P}_{25}-\mathrm{P}_{75}=5.0-10.0\right)$, and $113(46.5 \%)$ in both scenarios with a median of 10 patients per week $\left(\mathrm{P}_{25}-\mathrm{P}_{75}=10.0-20.0\right)$.

The geographical distribution of the completed questionnaires was as follows: Madrid (31.9\%), Andalusia (15.0\%), Catalonia (12.4\%), Valencian Community (7.1\%), Basque Country (7.1\%),
Castile-Leon (6.2\%), Galicia (6.2\%), and others $(<5 \%)$ including Aragon, Cantabria, Castile-La Mancha, Balearic Islands, Canary Islands, and Murcia.

\section{Results}

The most frequent reason for consultation was AGA (38\%), followed by telogen effluvium (24\%), alopecia areata (12\%), frontal fibrosing alopecia (FFA, 12\%), lichen planopilaris (5\%), folliculitis decalvans (4\%), and others (5\%). Statistically significant differences were found between the reasons for consultation in public ver- 
Fig. 3. Frequency of each treatment in patients with postmenopausal female androgenic alopecia (blue bar: public activity; orange bar: private activity). PRP, plateletrich plasma.

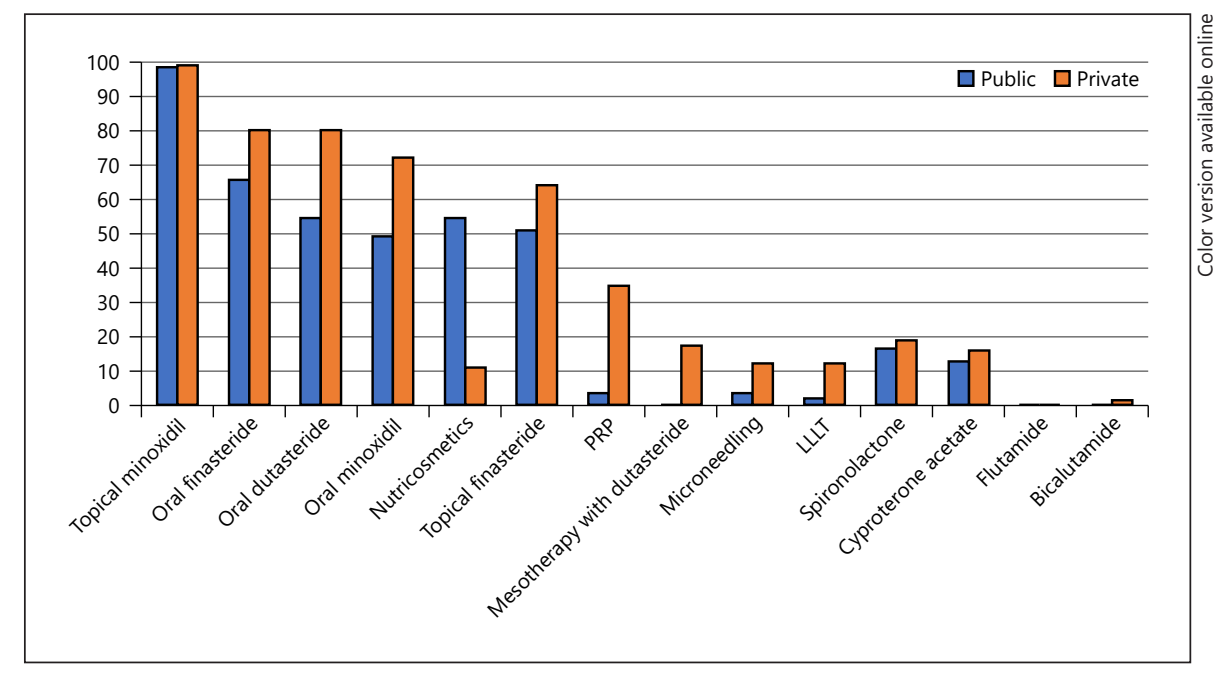

sus private system for telogen effluvium (18 vs. $23 \%$; $p=$ 0.037 ), alopecia areata ( 14 vs. $9 \%$; $p=0.001$ ), FFA (14 vs. $9 \% ; p=0.005$ ), lichen planopilaris (5 vs. $3 \% ; p=0.025$ ), folliculitis decalvans ( 3 vs. $2 \% ; p=0.007$ ), discoid lupus erythematosus ( 3 vs. $2 \% ; p=0.044$ ), and other alopecia (2 vs. $4 \% ; p=0.036)$. In terms of gender distribution among patients with AGA, statistically significant differences were found in the proportion of men between public versus private assistance ( 30 vs. $40 \%$; $p=0.002$ ).

Prescribing habits for male AGA are shown in Figure 1. Statistically significant differences were found between public versus private prescription for oral minoxidil (40 vs. $58.7 \% ; p=0.035$ ), platelet-rich plasma (PRP) (3.6 vs. $28 \% ; p=0.000$ ), dutasteride microinjections ( 0 vs. $16 \%$; $p=0.002$ ), microneedling ( 3.6 vs. $14.7 \% ; p=0.039$ ), and LLLT ( 1.8 vs. $10.7 \% ; p=0.05$ ).

Treatments used in premenopausal women with AGA are represented in Figure 2. Statistically significant differences were found between public versus private prescription for topical finasteride ( 36.4 vs. $60 \% ; p=0.008$ ), oral finasteride ( 32.7 vs. $50.7 \%$; $p=0.042$ ), PRP (5.5 vs. $36 \%$; $p=0.000$ ), microneedling ( 3.6 vs. $16 \% ; p=0.025$ ), dutasteride microinjections ( 0 vs. $12 \% ; p=0.008)$, and LLLT ( 1.8 vs. $10.7 \% ; p=0.050)$.

Treatments prescribed in postmenopausal AGA are shown in Figure 3. Statistically significant differences were found between public versus private prescription for oral minoxidil ( 49.1 vs. $72 \%$; $p=0.008$ ), oral dutasteride (54.5 vs. $80 \% ; p=0.002)$, PRP (3.6 vs. $34.7 \% ; p=0.000$ ), dutasteride microinjections ( 0 vs. $17.3 \%$; $p=0.001$ ), microneedling (3.6 vs. $12 \% ; p=0.025)$, and LLLT (1.8 vs. $12 \% ; p=0.032)$.

Treatments Prescribed for Androgenic Alopecia in Spain in 2019
Regarding hair transplantation, only $4.9 \%$ of the dermatologists who responded to the survey performed surgical transplantation. Last, trichology training during the residency was insufficient for $52.6 \%$ of the respondents.

\section{Discussion}

If we compare these results with those of 2017, AGA was the most frequent reason for consultation, followed by telogen effluvium and alopecia areata. The most frequent scarring alopecia was FFA again. A common finding is that there is an increase in the prescription of oral dutasteride in the 3 types of AGA: in male AGA, from 33 to $75.7 \%$ currently; in premenopausal AGA, from 20 to 41.2\%; and in postmenopausal AGA, from 35 to $71.6 \%$ [1].

Another observation is the irruption of oral minoxidil among the most used treatments, being prescribed in male AGA by $50.6 \%$ of the dermatologists who answered the questionnaire, in premenopausal AGA by $67.9 \%$, and in postmenopausal AGA by $63 \%$. Despite its off-label use, the scientific evidence for this treatment is increasing [2-4].

Conversely, there is a decrease in the prescription of cyproterone acetate from 58 to $42.4 \%$ and from 16 to $13.2 \%$ in premenopausal and postmenopausal AGA, respectively [1]. However, bicalutamide appears as a novel antiandrogen treatment in premenopausal AGA [5].

In private system, mesotherapy with dutasteride can complement oral androgen treatment or apply it as monotherapy in patients with intolerance, contraindications, or who do not want to use oral antiandrogens [6]. 
In conclusion, AGA represents the most frequent alopecia in the trichology clinic. The most frequently prescribed treatments in male AGA and postmenopausal AGA by dermatologists in Spain were topical minoxidil and oral finasteride, while in premenopausal AGA, they were topical minoxidil, oral contraceptives, and nutricosmetics. Over the past 3 years, the prescription of oral dutasteride has increased drastically and oral minoxidil has appeared among the most prescribed therapies for male, premenopausal, and postmenopausal AGA.

\section{Acknowledgement}

The authors thank all dermatologists who have contributed with their answers.

\section{Statement of Ethics}

The authors declare that the research was conducted ethically in accordance with the World Medical Association Declaration of Helsinki. Ethics approval was not required.

\section{Conflict of Interest Statement}

The authors declare no conflicts of interests.

\section{Funding Sources}

The authors did not receive any funding.

\section{Author Contributions}

C.P.-O.: design of the work, acquisition, analysis and interpretation of data, writing, final approval, and agreement to be accountable for all aspects of the work. D.S.-C.: analysis and interpretation of data, design of the work, critical revision, final approval, and agreement to be accountable for all aspects of the work. D.F.-N.: acquisition of data, critical revision, final approval, and agreement to be accountable for all aspects of the work. J.J.-C.: acquisition of data, critical revision, final approval, and agreement to be accountable for all aspects of the work. D.O.-Q.: acquisition of data, critical revision, final approval, and agreement to be accountable for all aspects of the work. O.M.M.-A.: analysis and interpretation of data, critical revision, final approval, and agreement to be accountable for all aspects of the work. S.V.-G.: design of the work, critical revision, final approval, and agreement to be accountable for all aspects of the work.

\section{References}

1 Pindado-Ortega C, Saceda-Corralo D, Buendía-Castaño D, Fernández-González P, Moreno-Arrones ÓM, Fonda-Pascual P, et al. Prescribing habits for androgenic alopecia among dermatologists in Spain in 2017: a cross-sectional study. Actas Dermosifiliogr. 2018 Aug;109(6):536-42.

2 Jimenez-Cauhe J, Saceda-Corralo D, Rodrigues-Barata R, Hermosa-Gelbard A, Moreno-Arrones OM, Fernandez-Nieto D, et al. Effectiveness and safety of low-dose oral minoxidil in male androgenetic alopecia. J Am Acad Dermatol. 2019;81(2):648-9.
3 Sinclair RD. Female pattern hair loss: a pilot study investigating combination therapy with low-dose oral minoxidil and spironolactone. Int J Dermatol. 2018 Jan;57(1):104-9.

4 Pirmez R, Salas-Callo C-I. Very-low-dose oral minoxidil in male androgenetic alopecia: a study with quantitative trichoscopic documentation. J Am Acad Dermatol. 2020 Jan; 82(1):e21-2.
5 Fernandez-Nieto D, Saceda-Corralo D, Rodrigues-Barata R, Hermosa-Gelbard A, Moreno-Arrones O, Jimenez-Cauhe J, et al Oral bicalutamide for female pattern hair loss: a pilot study. Dermatol Ther. 2019 Nov;32(6): e13096. Epub 2019 Oct 10.

6 Saceda-Corralo D, Rodrigues-Barata AR, Vañó-Galván S, Jaén-Olasolo P. Mesotherapy with dutasteride in the treatment of androgenetic alopecia. Int J Trichology. 2017;9(3) $143-5$. 